Revue des patrimoines

$4 \mid 2004$

Les réseaux de la villégiature

\title{
Les références culturelles des émigrants mexicains de la vallée de Barcelonnette : du grand magasin à la villa
}

Hélène Homps

(2) OpenEdition

Journals

Édition électronique

URL : http://journals.openedition.org/insitu/2236

DOI : 10.4000/insitu.2236

ISSN : 1630-7305

Éditeur

Ministère de la Culture

Référence électronique

Hélène Homps, « Les références culturelles des émigrants mexicains de la vallée de Barcelonnette : du grand magasin à la villa », In Situ [En ligne], 4 | 2004, mis en ligne le 19 avril 2012, consulté le 07

septembre 2020. URL : http://journals.openedition.org/insitu/2236 ; DOI : https://doi.org/10.4000/ insitu.2236

Ce document a été généré automatiquement le 7 septembre 2020.

\section{cc) $(1) \&$}

In Situ Revues des patrimoines est mis à disposition selon les termes de la licence Creative Commons Attribution - Pas d'Utilisation Commerciale - Pas de Modification 4.0 International. 


\title{
Les références culturelles des émigrants mexicains de la vallée de Barcelonnette : du grand magasin à la villa
}

\author{
Hélène Homps
}

1 C'est au Mexique où ils émigrent massivement entre 1850 et 1950, alors qu'ils détiennent le monopole du commerce et de l'industrie textile ${ }^{1}$, que les émigrants de la vallée de Barcelonnette font l'expérience de la modernité et découvrent le langage de l'architecture et son pouvoir de représentation. Acteurs du siècle si justement baptisé « siècle de l'Industrie » (François Loyer) dans la jeune république fédérative des Etatsunis du Mexique (principalement sous le gouvernement de Porfirio Diaz, 1876-1911), ils deviennent les promoteurs d'une nouvelle architecture urbaine monumentale liée à la création d'un programme spécifique : celui du grand magasin. Du grand magasin à la villa édifiée de retour du Mexique, les références culturelles des anciens émigrants « mexicains $»^{2}$ sont les mêmes, directement issues de l'art industriel contemporain.

\section{El afrancesamiento, I'influence française}

Dans le Mexique du Porfiriato, où l'influence française, el afrancesamiento devient le véhicule privilégié de la modernité, «el modelo civilizador que todo lo incluye: la literatura, la musica, la poesia, pero tambien las modernas fabricas, los grandes almacenes, las modas y el arte del bien vivir ${ }^{3}$, l'architecture urbaine et le décor monumental suivent de près les modèles fournis par un éclectisme venu d'Europe et particulièrement de Paris. Les bâtiments officiels, les bâtiments publics et commerciaux parmi lesquels les grands magasins de nouveautés édifiés par les émigrants de la vallée de Barcelonnette multiplient les références à cette architecture "post-baroque » qui habille les capitales européennes et les sites consacrés à la villégiature. De la même façon, l'urbanisme des nouveaux quartiers suburbains, les modernes colonias, loties 
pour une clientèle étrangère aisée où prédomine el estilo francès actual ${ }^{4}$ portent la marque d'une nouvelle vision néo-classique et européenne qui l'emporte sur la vision coloniale et préhispanique, héritage de la conquête espagnole ${ }^{5}$.

\section{La référence au modèle parisien}

Figure 1

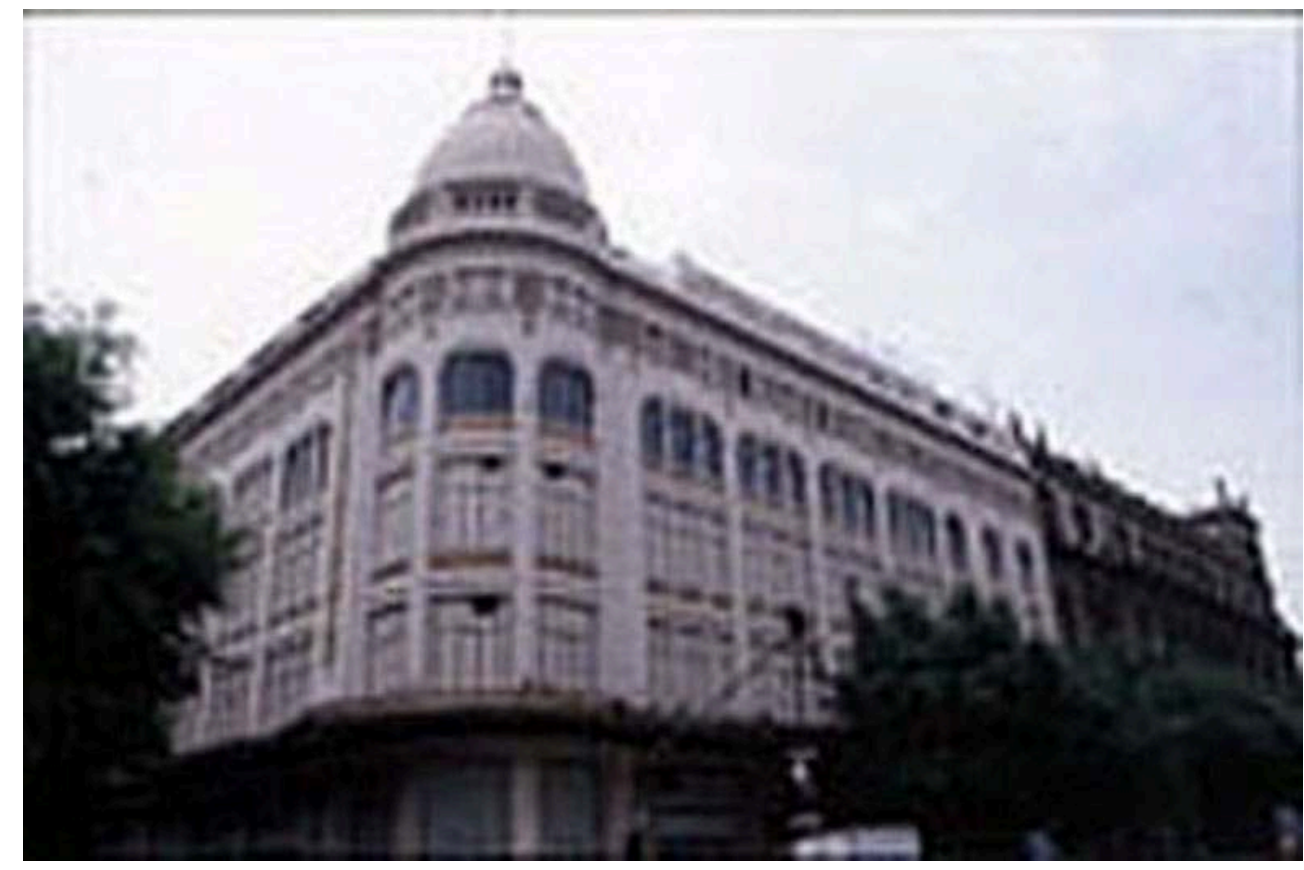

Grand magasin EL PALACIO DE HIERRo à Mexico

Phot. H. Homps () Musée de la Vallée, Barcelonnette, 2001

3 Calqués sur leurs aînés parisiens et construits avec les matériaux importés des ateliers et manufactures françaises, les nouveaux établissements de nouveautés qui nous occupent ici, édifiés, la plupart du temps, au cœur du noyau historique des principales villes mexicaines, exhibent tous « une écriture on ne peut plus post-haussmanienne de dômes et de grands combles cintrés dont la protubérance accentue la majesté des volumes, silhouettés à chaque carrefour $»^{6}$. Les nouveaux "monuments» de la république mexicaine sont l'œuvre d'architectes français, principalement parisiens, invités par le gouvernement mexicain pour construire les bâtiments officiels de $\mathrm{Mexico}^{7}$. Le magasin-fondateur, baptisé de façon emblématique Palacio de Hierro (Palais de fer) (fig. $\mathbf{n}^{\circ} \mathbf{1}$ ) voit le jour à Mexico, en 1891, édifié par les frères Jules et Joseph Tron. Dessiné sur les plans de l'architecte parisien Geoges Debrie associé à l'ingénieur Georges Pierron, il adopte pour la première fois au Mexique une ossature de fer directement inspirée par les prouesses technologiques de la tour récemment construite à Paris par l'ingénieur Eiffel. 
Figure 2

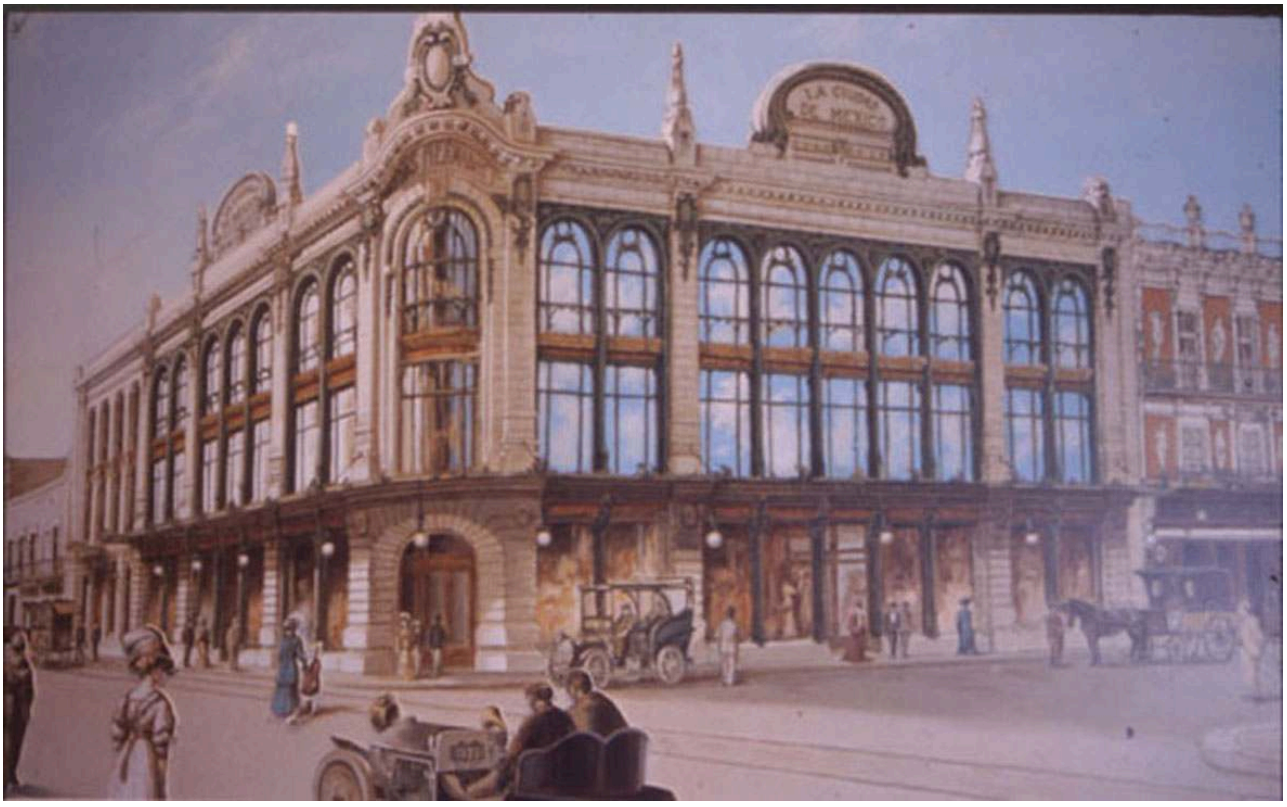

La « Ciudad de Mexico » à Puebla, 1895, lithographie

Phot. H. Homps @ Musée de la Vallée, Barcelonnette, 2001

4 Avec Las Fabricas Universales, édifiées à proximité par Alexandre Reynaud, l'architecte parisien Eugène Ewald reprend le modèle du grand magasin avec rotonde couronnée d'un dôme. Dans sa monographie intitulée précisément Un Edificio Francés, Leticia Gamboa met l'accent sur le caractère inédit de ce langage architectural qui distingue, à Puebla, le grand magasin La Ciudad de Mexico (fig. n $^{\circ}$ 2), construit en 1895 par les frères Lions et librement « inspirado en el modelo de la Samaritaine, una de las tiendas mas renombradas de Francia $»^{8}$. 


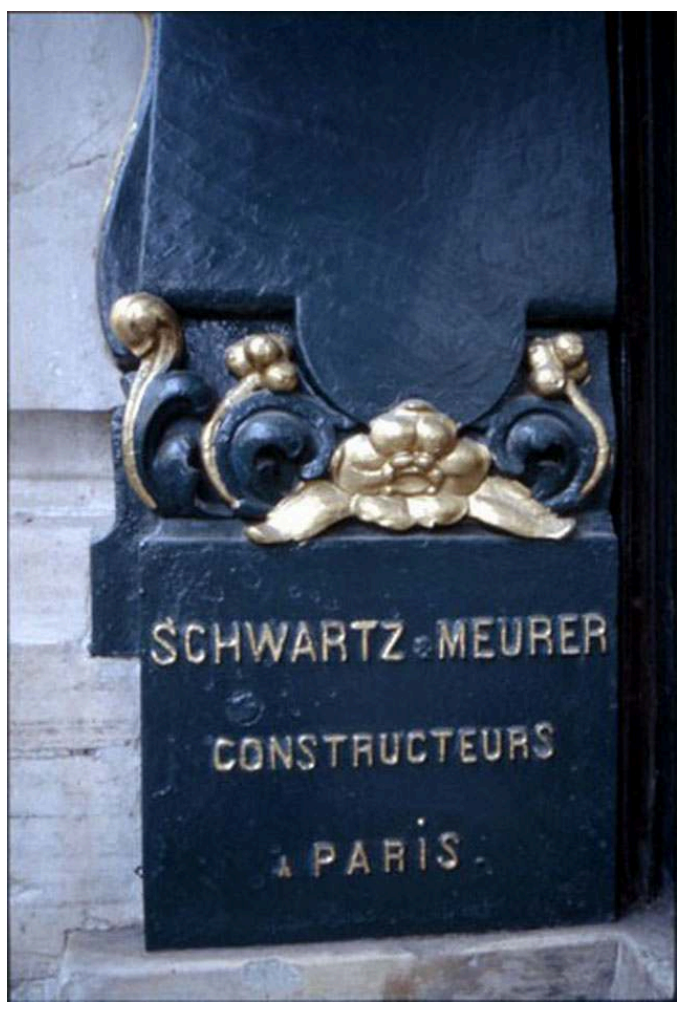

La « Ciudad de Mexico » à Puebla, 1895 ; constructeurs : Schwartz et Meurer, Paris Phot. H. Homps (c) Musée de la Vallée, Barcelonnette, 2001

5 Son ossature métallique comme les pierres de taille proviennent directement de France. Les pilastres extérieurs portent ainsi la signature des constructeurs Schwartz et Meurer, Paris (fig. $\mathbf{n}^{\circ}$ 3). A Guadalajara, l'architecte Ernesto Fuchs imprime de la même façon le « style français » à l'imposante façade de Las Fabricas de Francia ${ }^{9}$.

Toutes ces cathédrales du commerce ne se contentent pas d'offrir les derniers articles au goût du jour, les plus importants d'entre-eux s'intéressent aussi à l'ameublement et à la décoration, fabriquant dans leurs ateliers, « un grand choix de tapis, carpettes, rideaux, tapisseries anciennes et Art nouveau, ainsi qu'un grand assortiment de meubles français, anglais, italiens et américains et s'engagent «à meubler et à décorer des maisons particulières, bureaux publics et commerciaux $»^{10}$. Cette culture industrielle centrée sur l'objet manufacturé se retrouve au cœur du projet architectural et décoratif de la villa édifiée par les anciens émigrants «mexicains » de retour dans leur vallée ${ }^{11}$.

\section{Le réseau des Maisons spécialisées}

Entièrement sélectionné sur catalogue, le décor de la villa (parquet, huisserie, cheminées, ferronnerie, décor papier peint etc.) est directement issu des procédés industriels contemporains et témoigne $d u$ "goût passionné de l'époque pour l'ornement ». Exceptionnellement conservé (et soigneusement relié), le mémoire de la construction révèle le nombre important de ces industries et manufactures consacrées à l'équipement et à la décoration de l'habitation moderne réparties sur le territoire 
national (Marseille, Nice, Gap, Lyon, Nancy, Paris) et toutes abondamment titrées (participation aux concours des expositions universelles).

Figure 4

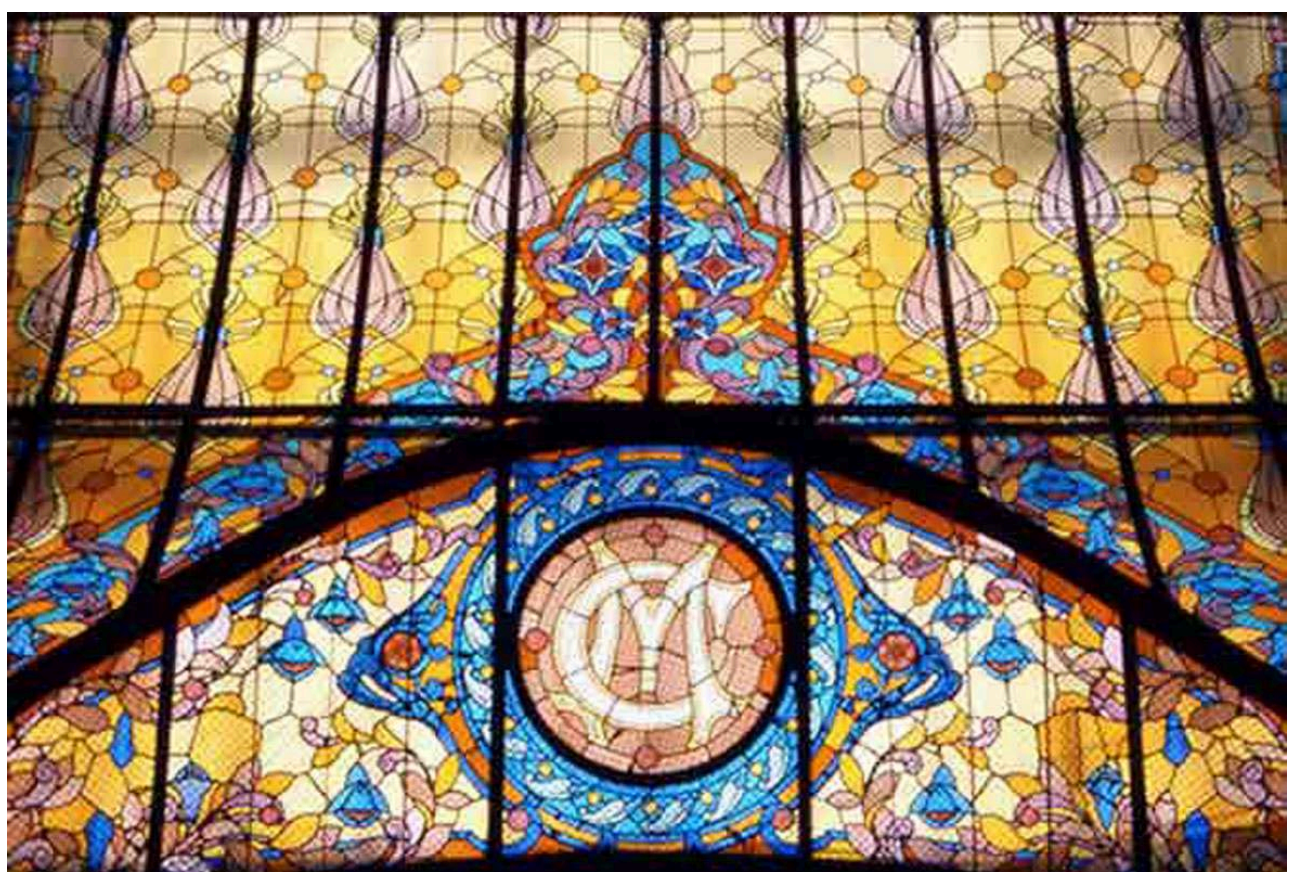

Verrière zénithale du magasin « Centro Mercantil » à Mexico, par Jacques Gruber (détail), aujourd'hui Grand Hôtel de la Ciudad de Mexico

Phot. H. Homps @ Musée de la Vallée, Barcelonnette, 2001

Autant de références directes à l'art industriel contemporain qui qualifie de la même façon les grands magasins, les sièges sociaux d'entreprise ou les grands hôtels (programme qui marquera l'activité des anciens négociants barcelonnettes retirés sur le littoral méditerranéen), « réduisant l'architecte au rôle de décorateur ». Dans cette vitrine dominée par la production décorative industrielle, l'intervention d'un artiste est exceptionnelle. C'est le cas du maître-verrier nancéien Jacques Gruber que l'on retrouve au Mexique et dans la vallée de Barcelonnette ${ }^{12}$ (fig. $\mathbf{n}^{\circ}$ 4).

\section{Primauté de l'héritage rationaliste}


Figure 5

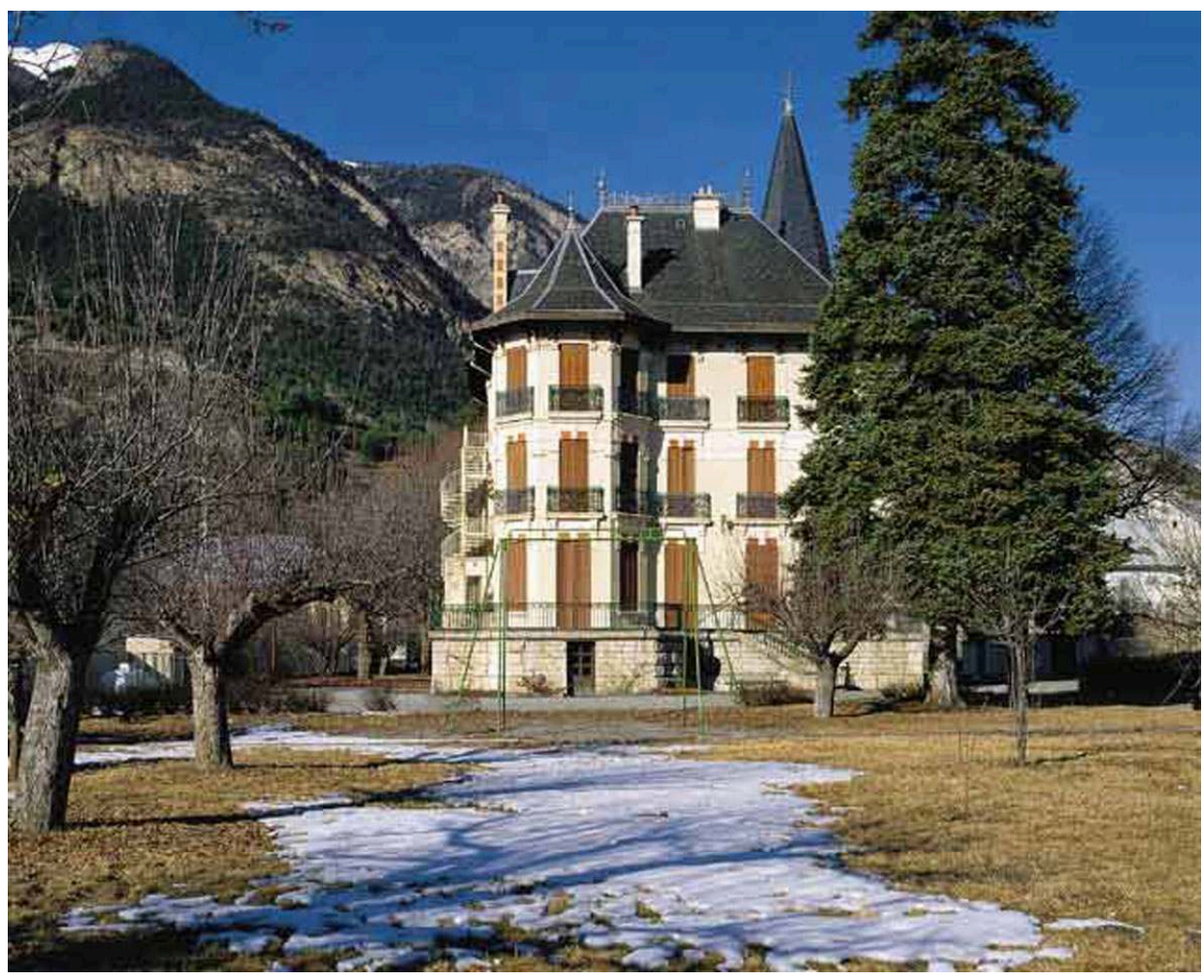

Villa Morélia à Jausiers, 1900. Elévation sud, sur parc

Phot. Inv. M. Heller (c) Inventaire général, ADAGP, 2001

9 Dans cet univers marqué par une culture matérielle, quelles références culturelles les architectes sollicités par cette ancienne clientèle assidue des « grandes fêtes populaires de l'industrie et de la consommation" vont-ils apporter et introduire sur les sites alpins de Barcelonnette et Jausiers (fig. $\mathbf{n}^{\circ}$ ) ? Après une période d'adaptation (1880-1890) durant laquelle, l'architecture des nouvelles constructions se contente de reproduire le modèle urbain et classique local, une nouvelle écriture éclectique et pittoresque signée Eugène Marx ${ }^{13}$, voit le jour ; elle va se révéler décisive dans la mise en place d'un répertoire de formes propres à séduire les anciens négociants pour lesquels «paraitre est la grosse affaire » (Viollet-le-Duc). Ainsi la grande majorité des nouveaux propriétaires, informés des modèles diffusés par les revues d'architecture contemporaines ${ }^{14}$, vont-ils se " reconnaître » dans l'indispensable effet-de-silhouette qui trouve dans les années 1890-1914, avec la création de la "villa-château », son plus parfait achèvement. Le choix d'un éclectisme tempéré prédomine qui ignore curieusement toutes références culturelles à une architecture exotique, néo-mauresque (les anciens émigrants ont pourtant fréquenté l'Algérie, l'Espagne et fait le voyage de l'Alhambra) ou pré-hispanique (tous ont côtoyé pendant vingt ans au Mexique les vestiges de la culture précolombienne). 
Figure 6

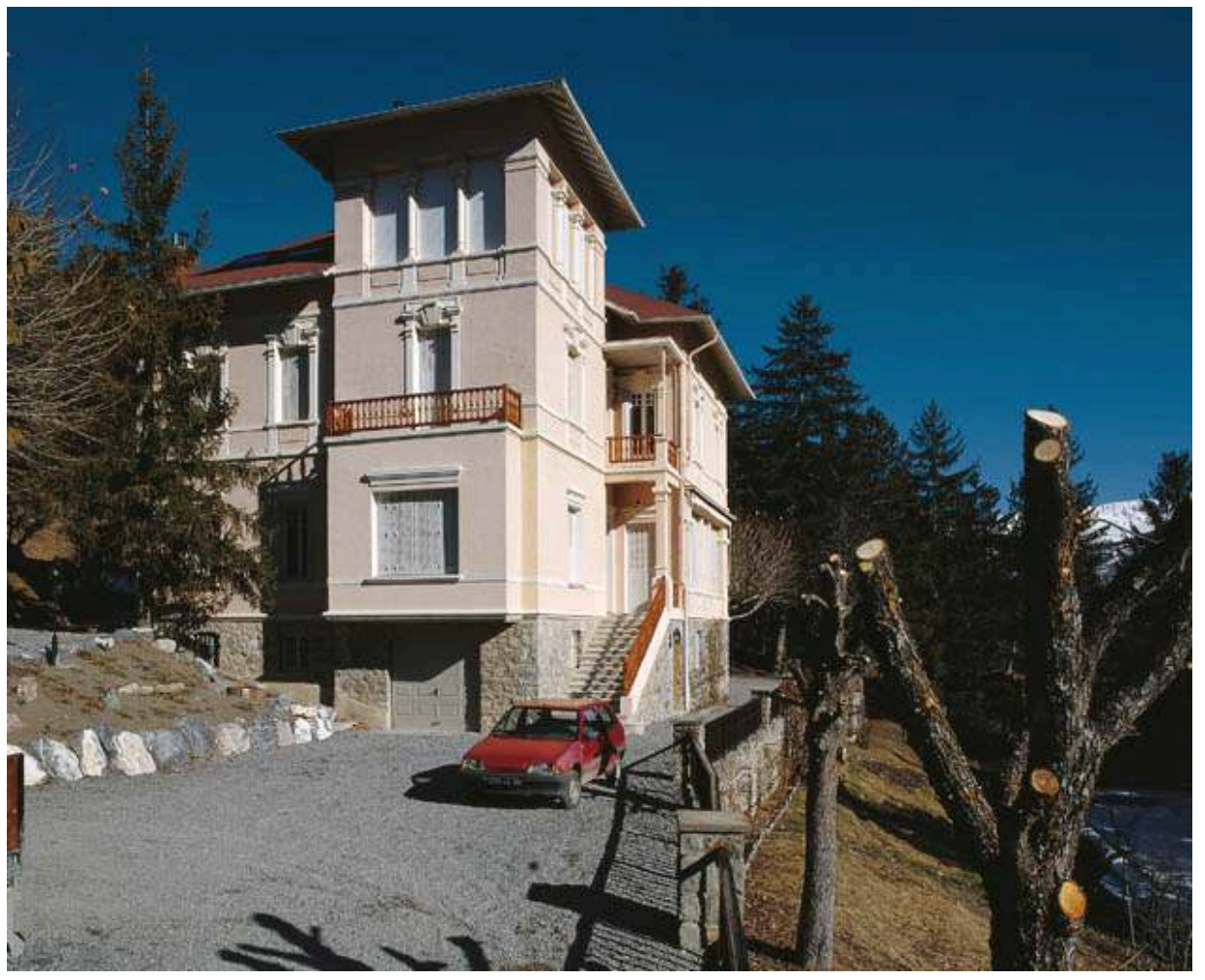

Villa Costebelle, 1913. Elévation sud-ouest, entrée et garage en soubassement

Phot. Inv. M. Heller @ Inventaire général, ADAGP, 2001

10 En rupture avec le langage rationaliste, seul l'architecte italien Ramelli, installé à Lugano, introduit dans les années 1910 une nouvelle référence culturelle fournie par l'Italie toute proche. Les nouveaux modèles affichent une écriture méditerranéenne empreinte d'italianisme qui trahit un intérêt inédit pour le site, propre à l'esprit de la villégiature (fig. $\mathbf{n}^{\circ}$ 6). Avec les années 1930 et la fin de la commande des villas dans la vallée, l'écriture «art déco» fait son apparition adaptée au programme d'une habitation désormais plus modeste. 
Figure 7

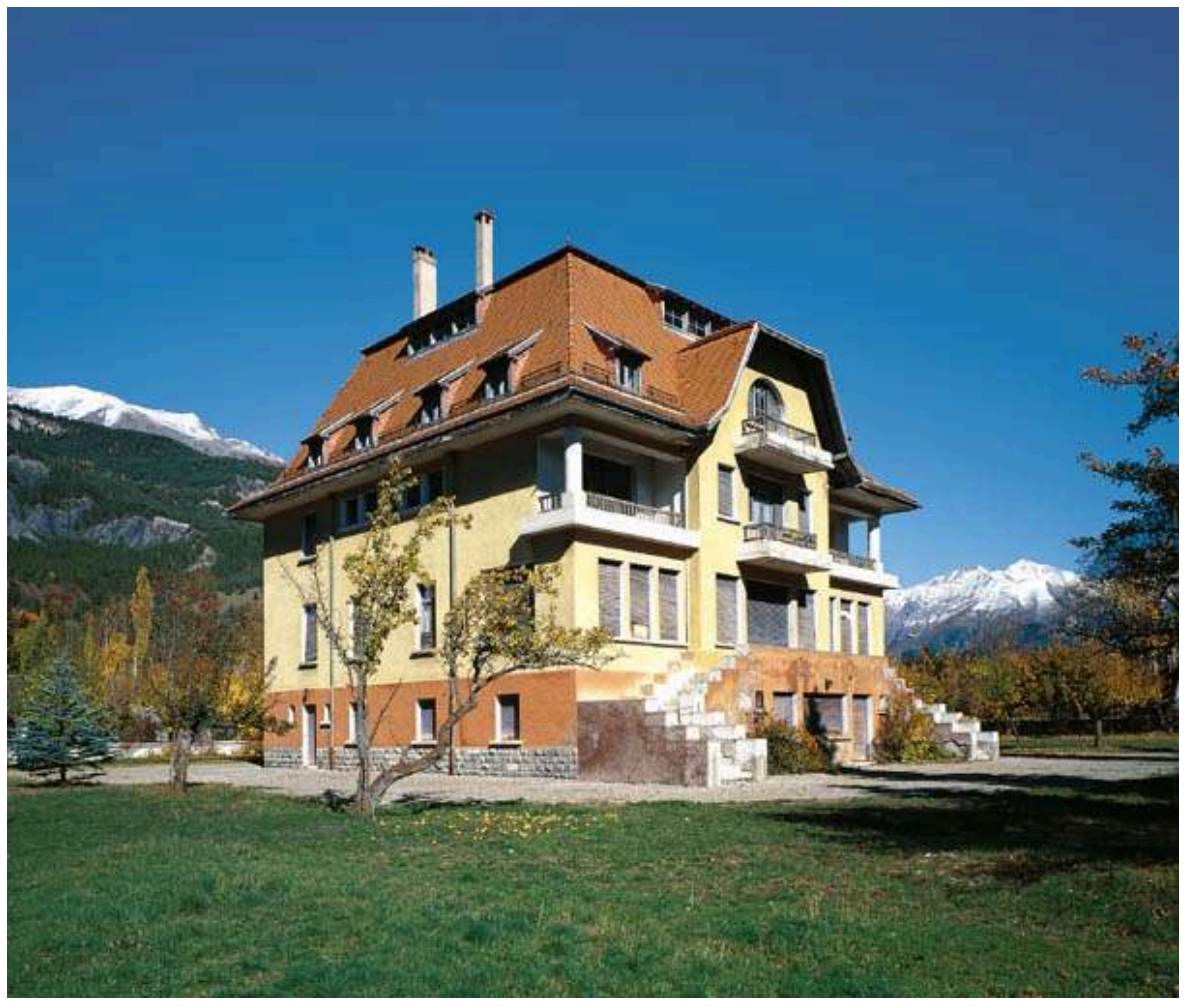

Villa Bleue, 1931. Elévation sud-ouest

Phot. Inv. M. Heller @ Inventaire général, ADAGP, 2001

11 Seule la grande villa construite pour le fondateur du magasin la Francia Maritima à Mexico, la Villa bleue (1931) (fig. $\mathbf{n}^{\circ}$ 7), rassemble autour de l'architecte basque Joseph Hiriart une équipe talentueuse d'architectes et artistes-décorateurs remarquée à l'Exposition internationale des arts décoratifs de 1925 à Paris, pour un programme exceptionnel.

\section{Conclusion}

Du grand magasin à la villa, les références culturelles restent les mêmes, bénéficiant parfois du même réseau (associant architectes, artistes décorateurs et maisons spécialisées), portées par une communauté de maîtres d'ouvrage soucieuse d'exprimer avant tout l'image du progrès et de la réussite sociale. Ainsi les villas de Barcelonnette et Jausiers ${ }^{15}$ "garderont-elles le souvenir d'une classe particulière de la société d'une époque » qui correspond à ce que Gérard Monnier a appelé l'idéal du citoyenpropriétaire ou du rentier de $1900^{16}$. 


\section{NOTES}

1. Dès la fin du XVIII ${ }^{e}$ siècle et le début du XIX ${ }^{e}$, la vallée de Barcelonnette, aux confins des Alpes et de la Haute-Provence est le creuset d'un important mouvement migratoire vers les Amériques, (Louisiane, Mexique et Argentine). Appelés les «barcelonnettes", les habitants de la vallée émigrés au Mexique vont s'implanter et travailler dans l'industrie (textile), le commerce (de tissus) et la banque.

2. Nom donné dans la vallée, aux anciens émigrants barcelonnettes de retour du Mexique, fortune faite. "Toi qu'en Amérique on appelait barcelonnette, à Barcelonnette on t'appelle Américain (ou mexicain)» dans Chabrand, Emile. De Barcelonnette au Mexique. Paris : Plon, 1893.

3. Perez-Siller, Javier. Presentacion. In: Mexico-Francia, memoria de una sensibilidad comun. Siglos XIX.XX. Benemérita Universidad Autonoma de Puebla, Colegio de San Luis A.C CEMCA, 1998. Un programme de recherches intitulé " Mexico-Francia, memoria de una sensibilidad comun, siglos XIX e $_{\text {- }}$ $X X^{e}$ ", réunit depuis 1995 chercheurs mexicains et français sur le thème de la mémoire commune de l'émigration.

4. "De todas partes se ven casas de dos pisos guarnecidas de piedras y coronades por las tipicas mansardes (en français dans le texte) tan cartacteristicas de las ciudades de Francia ».In " Mexico en el Centenario de su indenpendencia 1810-1910«, in Album grafico de la républica Mexicana. 1910. Editado por el gran Establecimiento Lito-Tipografico de Müller Hermanos. Mexico. DF.

5. Fernandez-Christlieb, Federico. La Influencia Francesa en el urbanismo de la ciudad de Mexico: 1775-1910. In : Mexico-Francia, memoria de una sensibilidad comun. Siglos XIX.XX. Benemérita Universidad Autonoma de Puebla, Colegio de San Luis A.C CEMCA, 1998. Pages 228-265.

6. Loyer, François. Le siècle de l'Industrie, 1789-1914. Paris ; Lausanne : Skira, 1983.

7. C'est le cas de l'architecte Paul Dubois associé à l'architecte Emile Bénard (1844-1929), Grand prix de Rome en 1867, invité à Mexico pour le projet de construction de la Chambre des députés. Certains architectes français choisiront de s'installer dans la capitale mexicaine et travailleront pour le compte des entrepreneurs barcelonnettes. Ainsi Paul Dubois réalisera plusieurs bâtiments pour les Barcelonnettes parmi lesquels le second Palacio de Hierro (1921-1928) et le siège de la Compagnie Industrielle d'Orizaba, la CIDO.SA.

8. Gamboa-Ojeda, Leticia. Un edificio Francés. Puebla La ciudad y sus monumentos. Instituto de Ciencias Sociales y Humanidades Benemérita Universidad Autonoma de Puebla. Puebla, Mexico, 1997. 24 pages. "En el porfiriato muchos elementos empleados en arquitectura y decoracion se importaban de Europea y en especial de Paris ». p 22.

9. Les premiers établissements édifiés par les Barcelonnettes («Centro Mercantil », "Puerto de Liverpool ", Palacio de Hierro » etc.), sous le Porfiriato (1876-1911) occupent une place d'honneur dans les albums officiels édités à l'occasion des célébrations de l'Indépendance du Mexique (1810-1910). La construction des grands magasins se poursuit jusqu'à nos jours avec l'édification des derniers établissements du groupe «Liverpool » (Liverpool, Monterrey, octobre 2001) et « Palacio de Hierro » (Puebla, octobre 2002).

10. Publicité en français du magasin «Ciudad de Londres ", à Mexico, année 1912. "Dans notre rayon de tapisserie et décoration, nous avons toujours un grand choix de tapis, carpettes, rideaux, tapisseries anciennes et Art nouveau etc. Un grand assortiment de meubles français, anglais, italiens et américains ».

11. Répartie de 1870 à 1935 , l'édification des villas de Barcelonnette (50 villas) et de Jausiers (20 villas) suit les variations de la prospérité économique des émigrants barcelonnettes implantés au Mexique. On distingue ainsi une première phase, celle de l'implantation (jusqu'en 1890, période à laquelle correspond une première génération de villas, une deuxième phase, celle des grands 
magasins (1890-1920) qui voit la création de la «villa-château », puis une troisième, entre les deux guerres, qui fait écho aux difficultés économiques du commerce et de l'industrie au Mexique et se traduit dans la vallée par l'arrêt progressif de la construction des villas.

12. Présent sur le chantier des grands magasins à Mexico («Centro Mercantil » et «Palacio de Hierro »), Jacques Gruber (1871-1936) intervient aussi à Barcelonnette et Jausiers pour d'anciens émigrants (villa Javelly et Villa bleue). On citera aussi les lithographies marouflées sur toile d'Alfons Mucha (1860-1939) et les bas-reliefs signés du sculpteur lorrain, Clodion (1738-1814), qui restent des acquisitions d'œuvres d'artistes isolées dans le décor de la villa, entièrement dominé par les maisons spécialisées dans le décor intérieur.

13. Ancien élève et successeur de Joseph-Marius Letz (1838-1890) et président de la Société des Architectes des Bouches-du-Rhône, Eugène Marx marque de son empreinte le paysage urbain de Barcelonnette et Jausiers. Son intervention capitale fixe dans la vallée l'archétype de la «villachâteau » dans laquelle vont se reconnaître et s'identifier les nouveaux propriétaires rentiers.

14. Ainsi les planches de la Revue de l'Architecture Usuelle, (Rivoalen Emile, publiée par «la librairie spéciale d'architecture Emile Thezard, éditeur à Dourdan, Seine-et-Oise ») circulent entre les villas.

15. Homps-Brousse, Hélène. Villas en Ubaye, retour du Mexique (Alpes-de-Haute-Provence)... Aix-en-Provence : Association pour le Patrimoine de Provence, 2002.

16. Monnier, Gérard. La culture du citoyen-propriétaire : la diffusion des idées sur l'architecture de l'habitation suburbaine en France de Viollet-le-Duc à l'Art Nouveau. In Colloque du centre méridional d'histoire sociale, des mentalités et des cultures. Les intermédiaires culturels. Aix-en-Provence : Université de Provence, 1978. P 491 à 502.

\section{INDEX}

Mots-clés : Barcelonnette, Mexique, Palacio de Hierro (Mexico), Jules Tron, Joseph Tron, Georges Debrie (architecte), Georges Pierron, Las Fabricas Universales, Alexandre Reynaud, Eugène Ewald (architecte), La Ciudad de Mexico (Puebla), les frères Lions, Schwartz et Meurer (Paris), Ernesto Fuchs, Las Fabricas de Francia (Guadalajara), Jacques Gruber, Jausiers, Eugène Marx, Ramelli, La Francia Maritima (Mexico), la Villa bleue (Mexico), Joseph Hiriart

\section{AUTEUR}

\section{HÉLÈNE HOMPS}

Conservatrice du Musée de la Vallée, Barcelonnette. musee.vallee@wanadoo.fr 